\title{
Oxygen Detection in Biological Aqueous Solutions using Positive Muon for Cancer Research
}

\section{Amba Datt Pant}

Journal of Nepal Physical Society

Volume 4, Issue 1, February 2017

ISSN : 2392-473X

Editors:

Dr. Gopi Chandra Kaphle

Dr. Devendra Adhikari

Mr. Deependra Parajuli

JNPS, 4 (1), 7-10 (2017)

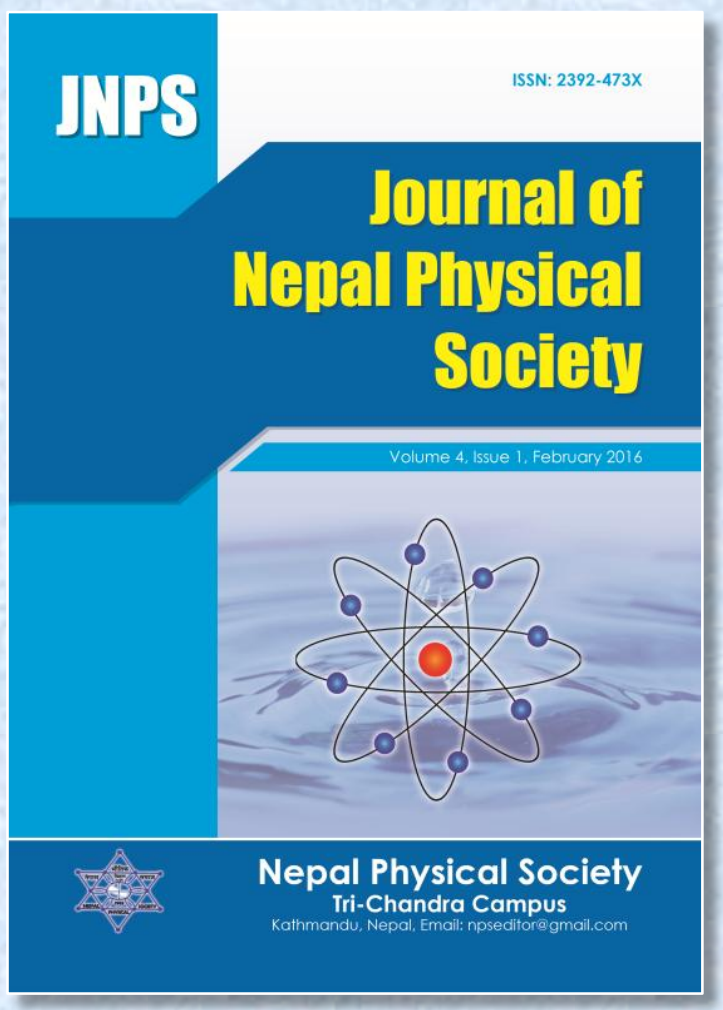

Published by:

Nepal Physical Society

P.O. Box : 2934

Tri-Chandra Campus

Kathmandu, Nepal

Email: npseditor@gmail.com 


\title{
Oxygen Detection in Biological Aqueous Solutions using Positive Muon for Cancer Research
}

\author{
Amba Datt Pant \\ Institute of Materials Structure Science, Muon Science Laboratory \\ High Energy Accelerator Research Organization (KEK), \\ 203-1 Shirakata, Tokai-mura, Naka-gun, Ibaraki 319-1106 Japan \\ Corresponding Email: pant@post.kek.jp
}

\begin{abstract}
Spin relaxation of muonium $(\mathrm{Mu})$ observed in molecular oxygen $\left(\mathrm{O}_{2}\right)$ dissolved in water shows characteristic behaviour which enables us to detect $\mathrm{O}_{2}$ in aqueous solution of albumin, serum and haemoglobin $(\mathrm{Hb})$. These results suggest development of a new method using positive muon to measure oxygen level in biological systems which can be applied for diagnosis and treatment of cancer.
\end{abstract}

Keywords: Hypoxia, Muonium, Asymmetry, Aqueous solutions, Biological molecule.

\section{INTRODUCTION}

Low oxygenation called hypoxia is known as an important factor in tumor biology and the response of tumors to treatment by radiation. Its measurement in specific region (tissue) of cancer patients may have an important predictive value in the management of treatment and outcome of the disease (Mancini et al., 1994; James et al., 1996; Kavanagh et al., 1999). The National Cancer Workshop (Tatum et al., 2006) on hypoxia imaging techniques pointed out the need of improved $\mathrm{O}_{2}$ detection methods for cancer treatment. Here, a new method using polarized positive muon $\left(\mu^{+}\right)$ produced at the accelerator facility is proposed to probe existence of the paramagnetic $\mathrm{O}_{2}$ in the cancer of eventually human body.

There are some existing techniques for this purpose with limitations (Tatum et al., 2006). For example; (a) PET 18F-labelled fluoro-misonidazole (18FFMISO) tracer is widely used in PET for hypoxia. It does not directly measure molecular $\mathrm{O}_{2}$ in the tumor, but the tracer retention affected primarily by $\mathrm{O}_{2}$. After radiation therapy, it images hypoxic cells and re-oxygenation. So it is like off-line technique.

(b) MRI Blood oxygen level-dependent (BOLD) MRI does not directly measure $\mathrm{O}_{2}$ molecule in blood but rather detects deoxy-Hb. During measurement we need to have special attention for use of the high magnetic fields for in-situ MRI. (c) EPR is another method which is not non-invasive.

Brown J. M. et al., (2004) mentioned that the concentration of oxygen in tumorous part in human body is heterogeneous (less than $5 \mathrm{mmHg}$ ). From muon spin measurements, the muon spin can probe oxygen in water (Jean et al., 1979; Nagamine and Nishiyama 1982; Roduner et al., 1995) between 0.3 to $300 \mathrm{mmHg}$ which perfectly matches to the range of hypoxia.

The proposed $\mathrm{Mu}$ relaxation method is able to detect and measure molecular $\mathrm{O}_{2}$ concentration in tissues directly. It can be used non-invasively in a small area of human body at any temperature and without any strong magnetic fields. So it will act as non-invasive sensitive needle with high spatial resolution.

Here, muon spin rotation and relaxation $(\mu S R)$ measurement on pure water, aqueous biological solutions of serum, albumin and hemoglobin under controlled $\mathrm{O}_{2}$ concentration is presented.

\section{EXPERIMENT}

In $\mu$ SR method, based on characteristic properties of positive muon-asymmetric decay and parity violation in weak interaction, decayed positrons from muons are collected by spectrometer around sample. Relation of asymmetry at any time can be written as difference between forward and backward counts as

$$
\text { Asymmetry }=\frac{F W-B W}{F W+B W} \times 100 \%
$$

Where FW is counts collected by forward counters and BW is that by backward counters. The time evolution of polarization (that is asymmetry) of an 
ensemble of muons implanted in a sample provides physical information about the interaction of the muon with its local environment. Muonium (bound state of $\mu^{+}$with electron) is like an light isotopes of hydrogen.

When energetic polarized positive muons are injected and stopped in water, these $\mu^{+}$take electronic states of diamagnetic $\mu^{+}$such as $\mu^{+} \mathrm{OH}$ with a fraction of $60 \%$, paramagnetic $\mathrm{Mu}$ with a fraction of $20 \%$ and a missing fraction of $20 \%$ (Jean et al., 1979; Nagamine and Nishiyama, 1982; Roduner et al., 1995). In the $\mathrm{Mu}$ fraction a half becomes ortho state with spin 1, providing a spin rotation signal with 100 times faster precession pattern with respect to the diamagnetic $\mu^{+}$. Spin rotation and its relaxation were detected under 2.1 $\mathrm{G}$ transverse fields. All the measurements were performed at room temperature.

The concentration of $\mathrm{O}_{2}$ is controlled in the sample and continuously circulated the sample to expose to muon beam. The experimental set up and the details about sample preparation can be found in (Pant, 2015; Pant et al., 2014; Nagamine et al., 2014). Measurements have been performed in aqueous biological samples of albumin, serum and hemoglobin. The $\mathrm{O}_{2}$ concentration in liquid is measured by commercially available NeoFox oxygen monitor which is calibrated as $\mathrm{O}_{2}$ concentration in gas phase being from $0 \%$ with $\mathrm{N}_{2}$ gas and $20.7 \%$ in air. Experiments were conducted at Port 2 of the RIKEN RAL muon facility in UK, using $60 \mathrm{MeV} / \mathrm{c}$ decay-in-flight polarized positive muons.

\section{RESULTS AND DISCUSSION}

The time spectra of asymmetry of $\mu \mathrm{SR}$ data observed in pure water with $\mathrm{O}_{2}$ concentration at less than $1 \%$ and $7.5 \%$ are presented in figure 1 (a) and (b), respectively. The Mu spin rotation signal (100 times larger frequency than that of $\mu^{+}$) superimposes with spin rotation of diamagnetic $\mu^{+}$. The spin precession of the $\mathrm{Mu}$ took faster relaxation against the increase of $\mathrm{O}_{2}$ concentration. These time spectra are fitted with function;

$F(t)=A_{\mu} \cos \left(\omega_{\mu} t+\phi_{\mu}\right)+A_{M u} \exp (-\lambda t) \cos \left(\omega_{M u} t+\right.$ $\left.\phi_{M u}\right)+B$

where $\mathrm{B}$ is the time-independent background and $\tau_{\mu}$ is the muon lifetime $(2.20 \mu s)$. The terms $A_{\mu}$ and $A_{M u}$ are the amplitudes of the spin precession corresponding to the polarization asymmetry for the $\mu^{+}$in diamagnetic states and in $\mathrm{Mu}$, respectively. The parameter $\lambda$ is the muonium relaxation rate while the relaxation rate of $\mu^{+}$in diamagnetic species is assumed to be negligible, the $\omega_{\mu}$ and the $\omega_{M u}$ are the muon and $\mathrm{Mu}$ precession frequencies, $\phi_{\mu}$ and $\phi_{M u}$ are the respective initial phases of their precessions.

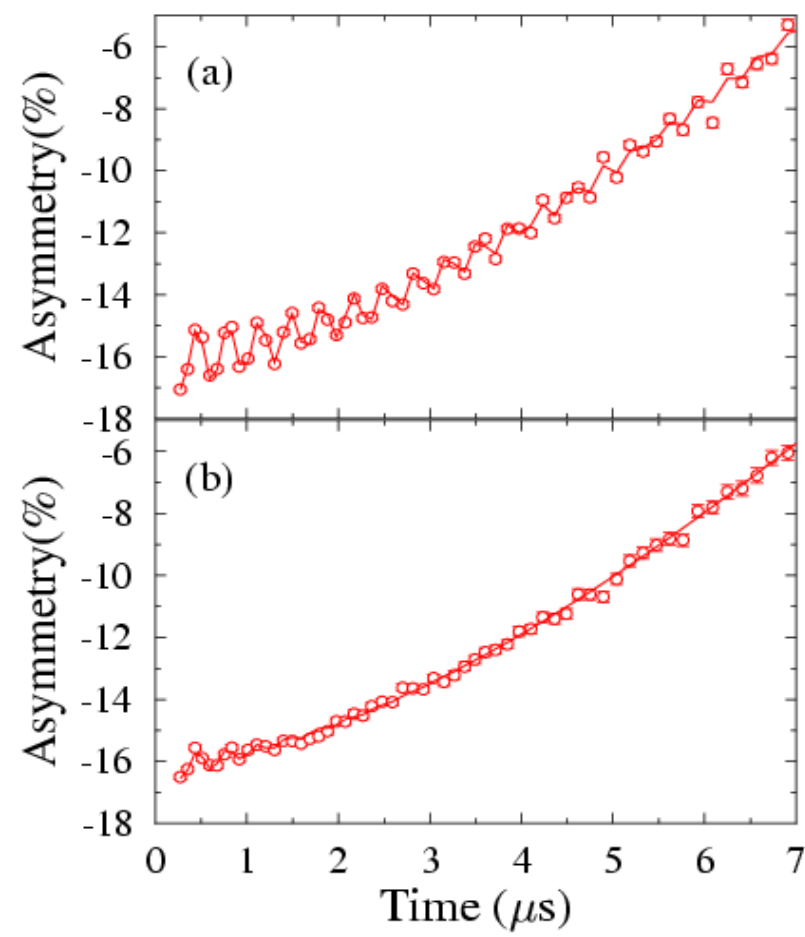

Fig. 1. Muon spin rotation time spectrum in water with $\mathrm{O}_{2}$ concentration (a) below $1 \%$ and (b) $7.5 \%$, at room temperature under $2.1 \mathrm{G}$ transverse field. Similar $\mathrm{O}_{2}$ dependence nature was observed in other biological aqueous solutions.

The relaxation rate of $\mathrm{Mu}$ with $\mathrm{O}_{2}$ concentration in pure water shows consistency with previous data (Roduner et al., 1995). Then the measurement was carried out in biological molecules. It is found that $\mathrm{Mu}$ exhibits similar relaxation change against the increase of $\mathrm{O}_{2}$ contents in albumin and serum aqueous solution as shown in figure 2. In albumin solution, the $\mathrm{Mu}$ takes stronger response to the increase of the $\mathrm{O}_{2}$ concentration. It suggests, in the presence of albumin, interaction between $\mathrm{Mu}$ and $\mathrm{O}_{2}$ becomes more active in higher concentration.

The $\mathrm{O}_{2}$ dependence $\mathrm{Mu}$ behavior in $0.5 \mathrm{~g} \mathrm{~L}^{-1} \mathrm{Hb}$ is presented in figure 2. Here, because of strong $\mathrm{O}_{2}$ absorption by deoxy- $\mathrm{Hb}$ (magnetic) to change it into oxy-Hb (nonmagnetic), it was expected the $\mathrm{Mu}$ relaxation takes a slower response against $\mathrm{O}_{2}$ increase in comparison with pure water and other biological aqueous solutions. The weakened response of $\mathrm{Mu}$ with increasing $\mathrm{O}_{2}$ concentration 
may be due to an influence of decreasing deoxy- $\mathrm{Hb}$ concentration with increasing dissolved molecular $\mathrm{O}_{2}$ concentration.

Based on behavior of oxygen in hemoglobin and $\mathrm{Mu}$ in pure water with increasing $\mathrm{O}_{2}$ concentration, a theoretical predication of the behavior of $\mathrm{Mu}$ has also done as below. For the qualitative understandings, it is assumed that the experimental data of $\mathrm{Mu}$ relaxation rate in aqueous solution of various $\mathrm{Hb}$ concentration and $\mathrm{O}_{2}$ concentration can be approximated in the simplest way as

$\lambda_{M \mu}=R_{l}(M u)+R_{2}(M u)$

The $R_{l}(M u)$ is relaxation rate of $\mathrm{Mu}$ due to deoxy$\mathrm{Hb}$ in solution, which is estimated using Hills equation, and the $R_{2}(\mathrm{Mu})$ is relaxation rate of $\mathrm{Mu}$ due to $\mathrm{O}_{2}$ in solution. In order to calculated $R_{2}(M u)$, both measurement in pure water and conversion of deoxy oxy-Hb taken into account. The result of theoretical estimation is presented in figure 3 (solid line is plot of equation 2 and marks are $\lambda$ of from equation 1). Theoretically it is easy to understand the minimum in the estimated curve which may be at the state of saturation. This behavior will be $\begin{array}{llll}\text { checked experimentally between } 2-5 \% & \mathrm{O}_{2}\end{array}$ concentrations. Furthermore, at less than $1 \% \mathrm{O}_{2}$, relaxation rate of $\mathrm{Mu}$ shows linear relation with the $\mathrm{Hb}$ concentration up to $2 \mathrm{~g} \mathrm{~L}^{-1} \mathrm{Hb}$ concentration.

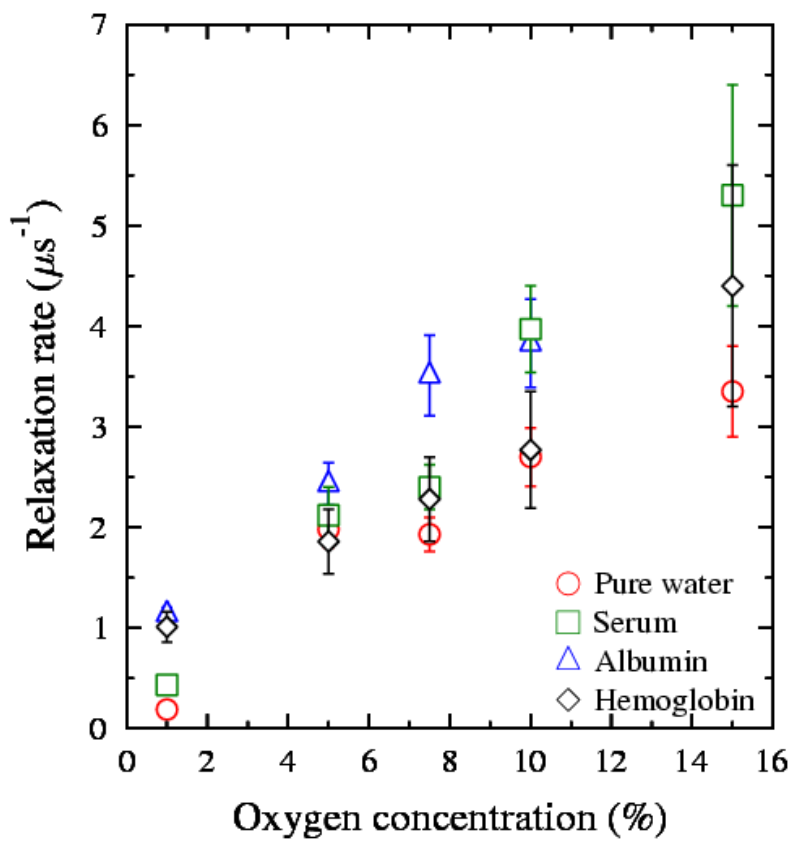

Fig. 2. Variation of relaxation rate of $M u$ with oxygen concentration in different biological aqueous solutions.

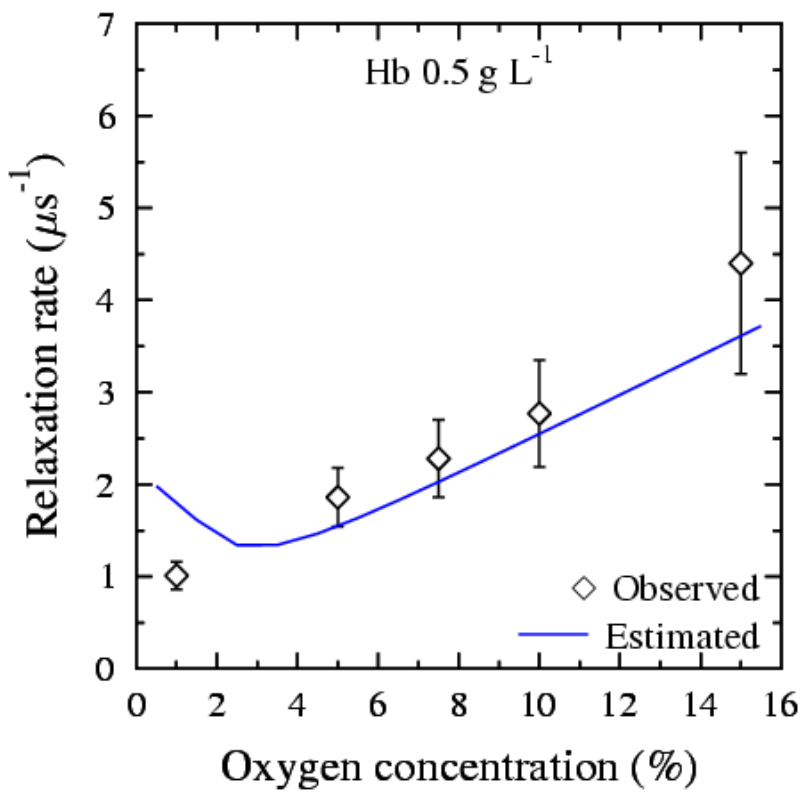

Fig. 3. Observed and estimated data of $\mathrm{O}_{2}$ concentration dependence of the Mu relaxation rate in $0.5 \mathrm{~g} \mathrm{~L}^{-1} \mathrm{Hb}$ aqueous solution.

\section{CONCLUSION AND FUTURE DIRECTIONS}

Concentration of molecular oxygen has successfully detected from the direct observation of muonium spin rotation signal in aqueous solution of albumin, serum and $\mathrm{Hb}$. The result is encouraging to apply the present method to a wide variety of the biological systems and at higher $\mathrm{Hb}$ concentration. Although measurements should be extended to more cases before clinical application, the most important case of the $\mathrm{Hb}$ aqueous solution was successfully studied which can be used for monitor of oxygen in human body $\left(\mathrm{O}_{2}\right.$ concentration in lung is $15 \%$ ). Before clinical application, since the biological system is complex, systematic studies using high spatial resolution muon micro beam of ultra slow muon (now under developing) in $\mathrm{J}$ PARC (Yashuhiro et al., 2014; Nagamine, 2014) will provide further idea about behavior of other magnetic molecules in human body and magnetism of blood.

\section{ACKNOWLEDGEMENTS}

I would like to thank Professors E. Torikai of University of Yamanashi; K. Nagamine of KEK, RIKEN, UCR Riverside; J. S. Schultz from University of California; K. Shimomura of KEK, W. Higemoto of JAEA and F. Pratt of ISIS, RAL for their fruitful discussion and every support. This work was supported by Grant-in-Aid for Scientific 
Research on Innovative Areas of the Ministry of Education, Culture, Sports, Science and Technology (MEXT) Japan, Grant Number 23108003 on Frontier of Materials, Life and Elementary Particles Science explored by Ultra Slow Muon (FY2011-2015).

\section{REFERENCES}

Brown, J. M. and Wilson W. R. (2004). Exploiting tumour hypoxia in cancer treatment. Nat Rev Cancer, 4(6): 437-47.

James, A.; Raleigh, M. W. D., and Donald, E. T. (1996). Measuring Tumor Hypoxia. Semin. Radiat. Oncol., 6: 37.

Jean, Y. C.; Fleming, D. G., and Walker, D. C. (1979). Reaction of muonium with $\mathrm{O}_{2}$ in aqueous solution. Chemical Physics Letters, 66(1): 187.

Kavanagh, M. C., et al. (1999). A Comparison in indivudula murine tumors of technique for measuring oxygen level. Int. J. Radiat. Oncol. Biol. Phys., 44: 1137.

Mancini, D. M., et al. (1994). In vivo magnetic resonance spectroscopy measurement of deoxymyoglobin during exercise in patients with heart failure. Demonstration of abnormal muscle metabolism despite adequate oxygenation. Circulation, 90(1):500-508.
Miyake, Y., et al. (2014). Ultra Slow Muon Project at J-PARC MUSE. JPS Conf. Proc., 2: 010101.

Nagamine, K., et al. (2014). RIKEN Accel. Prog. Rep., 47: 262.

Nagamine, K., and K. Nishiyama (1982). Longlived muonium in water revealed by pulsed muons. Chemical Physics Letters, 87(2): 186.

Nagamine, K. (2014). Past, present and future of ultra-slow muons. JPS Conf. Proc., 2: 010001.

Pant, A. D. (2015). Doctoral thesis submitted to Univerity of Yamanashi, Japan.

Pant, A. D., et al. (2014). Muonium response to oxygen content in biological aqueous solutions for cancer research. Journal of Physics: Conference Series, 551: 012043.

Roduner, E., et al. (1995). Effect of mass on particle diffusion in liquids studied by electron spin exchange and chemical reaction of muonium with oxygen in aqueous solution. Journal of the Chemical Society, Faraday Transactions, 91(13): 1935.

Tatum, J. L., et al. (2006). Hypoxia: importance in tumor biology, noninvasive measurement by imaging, and value of its measurement in the management of cancer therapy. Int $J$ Radiat Biol, 82(10): 699-757. 